Z. klin. Chem. u. klin. Biochem.

8. Jg., S. 420-424, Juli 1970

\title{
Vergleichende Untersuchung dẹs Mucopolysaccharid- und Kollagengehaltes in verschiedenen topographischen Zonen der Niere von Ratte, Hund und Schwein
}

\author{
Von H. Kresse und AnNemarie Grossmann \\ (Aus dem Institut für Physiologische Chemie der Universität Münster \\ und der Elektronenmikroskopischen Abteilung des Senckenbergschen Patbologischen Instituts der Universität Frankfurt|M.)
}

(Eingegangen am 24. März 1970)

1. Aus der Nierenpapille, unterteilt in Spitze, unteren und oberen Anteil des inneren Markes, dem äußeren Nierenmark und der Nierenrinde von Hund, Ratte und Schwein wurden saure Mucopolysaccharide (saure Glykosaminoglykane) präparativ dargestellt und Hexosamin-, Kollagen- und DNA-Gehalt des Gewebes bestimmt.

2. Der Mucopolysaccharidgehalt ist bei allen Tierarten im unteren Anteil des inneren Markes am größten (Hund: $2,7 \mathrm{mg} / \mathrm{g}$ Frischgewebe, Ratte: $1,3 \mathrm{mg} / \mathrm{g}$ Frischgewebe, Schwein: 1,6 mg/g Frischgewebe). Zur Nierentinde hin erfolgt eine stete Konzentrationsabnahme. In der Nierentinde lassen sich an Mucopolysacchariden lediglich $0,4 \mathrm{mg}$ (Hund) bzw. 0,3 mg (Ratte) und 0,5 mg (Schwein) pro Gramm Frischgewebe nachweisen.

An einzelnen Mucopolysaccharidtypen finden sich Hyaluronat, Chondroitinsulfat, Heparitinsulfat und Dermatansulfat. Heparitinsulfat und Dermatansulfat kommen in allen Nierenzonen vor. Hyaluronat ist in der Papillenspitze der Rattenniere nicht nachzuweisen. Chondroitinsulfat fehlt in der Nierentinde aller Tierarten.

3. Der Kollagengehalt steigt bei Hund und Schwein von der Papillenspitze (Hund: $23 \mathrm{mg} / \mathrm{g}$ Frischgewebe, Schwein: $15 \mathrm{mg} / \mathrm{g}$ Frischgewebe) bis zum oberen Anteil des inneren Markes auf Werte von $32 \mathrm{mg} / \mathrm{g}$ Frischgewebe (Hund) bzw. $20 \mathrm{mg} / \mathrm{g}$ Frischgewebe (Schwein) und fällt zur Rinde wieder stark ab (Hund: $3 \mathrm{mg} / \mathrm{g}$ Frischgewebe, Schwein: $4 \mathrm{mg} / \mathrm{g}$ Frischgewebe). Der Kollagengehalt der Rattenniere beträgt in allen Zonen etwa $2 \mathrm{mg} / \mathrm{g}$ Frischgetwebe.

4. Die DNA-Konzentration erreicht bei Ratte und Schwein ein Maximum im oberen Anteil des inneren Markes (Ratte: 6,3 mg/g Frischgewebe, Schwein: 5,6 mg/g Frischgewebe). Beim Hund liegt der höchste DNA-Gehalt im äußeren Mark (4,7 mg/g Frischgewebe). Die niedrigsten Werte werden in der Papillenspitze gemessen. Der DNA-Gehalt der Rattenniere ist mit Ausnahme der Papillenspitze höher als bei Hund und Schwein.

5. Eine eindeutige Korrelation zwischen Mucopolysaccharidgehalt in der Nierenpapille und Harnkonzentrierungsvermögen läßt sich auch bei Berücksichtigung des Verhältnisses von intra- und extrazellulätem Kompartiment nicht feststellen.

\section{Comparative studies of the mucopolysaccharide and collagen content in different topographical zones of the kidney of rat, dog and pig}

1. Acidic mucopolysaccharides (acidic glycosaminoglycans) were isolated preparatively from the kidney papillae separated into their apices, lower and upper part of the inner medulla, the outer kidney medulla and the kidney cortex of dog, rat and pig. The hexosamine, collagen and DNA contents of these tissues were determined.

2. In all the animal species, the highest mucopolysaccharide content was found in the lower part of the inner medulla (dog: $2.7 \mathrm{mg} / \mathrm{g}$ fresh tissue, rat: $1.3 \mathrm{mg} / \mathrm{g}$ fresh tissue, pig: $1.6 \mathrm{mg} / \mathrm{g}$ fresh tissue). In the kidney cortex only $0.4 \mathrm{mg}$ (dog), $0.3 \mathrm{mg}$ (rat) and $0.5 \mathrm{mg}$ (pig) of mucopolysaccharides per gram fresh tissue were detected. The individual mucopolysaccharide types found were hyaluronate, chondroitin sulphate, hepatitin sulphate and dermatan sulphate. Heparitin sulphate and dermatan sulphate occur in all zones of the kidney. Hyaluronate could not be detected in the apices of the papillae. Chondroitin sulphate was absent from the kidney cortex of all three species.

3. In dog and pig the collagen levels were $23 \mathrm{mg} / \mathrm{g}$ fresh weight (dog) and $15 \mathrm{mg} / \mathrm{g}$ fresh weight (pig) in the papillae apices, increasing to values of 32 and $20 \mathrm{mg} / \mathrm{g}$ fresh weight respectively in the upper part of the inner medulla and falling markedly again in the cortex to 3 and $4 \mathrm{mg} / \mathrm{g}$ fresh weight respectively. In all zones of the tat kidney, the collagen content was about $2 \mathrm{mg} / \mathrm{g}$ fresh tissue.

4. The DNA concentration in the rat and pig kidney was highest in the inner medulla (rat: $6.3 \mathrm{mg} / \mathrm{g}$ fresh tissue, pig: $5.6 \mathrm{mg} / \mathrm{g}$ fresh tissue). In the dog the highest DNA content was in the outer medulla $(4.7 \mathrm{mg} / \mathrm{g}$ fresh tissue). The lowest values were measured in the papillae apices. With the exception of the papillae apices, the DNA content of rat kidney is higher than in dog and pig.

5. No correlation can be established between the mucopolysaccharide content of the kidney papillae and the ability to concentrate the urine, even when the ratio of the intracellular and extracellular compartment is taken into consideration.

Während in der Nierenrinde Tubuli und Blutkapillaren eng aneinander grenzen, liegt im Nierenmark eine zur Papillenspitze hin immer breiter werdende interstitielle Grundsubstanz zwischen tubulären und kapillären Basalmembranen $(1,2)$. Diese extrazelluläre Grundsubstanz gibt vor allem im Nierenmark eine positive histochemische Reaktion auf saure Mucopolysaccharide (3-5).
Saure Mucopolysaccharide, die in nativem Zustand stets kovalent mit Protein verknüpft sind, sind als Polyanionen in der Lage, Kationen und Wasser $(6,7)$ reversibel zu binden. Das Wasserbindungsvermögen wird zwar in Gegenwart von Elektrolyten deutlich reduziert, doch beträgt das effektive hydrodynamische Volumen eines Chondroitinsulfat-Proteins noch $100-150 \mathrm{~m} l$ $0,15 \mathrm{M} \mathrm{NaCl} / \mathrm{g}$. Der Gedanke liegt daher nahe, sauren 
Mucopolysacchariden eine Rolle bei der Harnkonzentrierung im Gegenstrom zuzuschreiben $(8,9)$, zumal noch unklar ist, wie die im äußeren Nierenmark durch aktiven Natriumtransport aus dem aufsteigenden Schleifenschenkel in Gang gesetzte Gegenstrommultiplikation im inneren Nierenmark fortgesetzt wird (10).

Ziel der vorliegenden Arbeit war deshalb, das Verteilungsmuster saurer Mucopolysaccharide in definierten Nierenabschnitten, nämlich in Papillenspitze, unterem Anteil des inneren Markes, oberem Anteil des inneren Markes, äußerem Mark und in der Nierenrinde bei verschiedenen Tierarten zu bestimmen, die in unterschiedlichem Ausmaß Harn zu konzentrieren vermögen. Für diesen Zweck schien die vergleichende Untersuchung der Nieren von Hund, Ratte und Schwein geeignet, da sich bei den genannten Tieren die Fähigkeit zur Harnkonzentrierung deutlich unterscheidet (maximale Osmolarität des Harns beim Hund $2000 \mathrm{mOsmol} / \mathrm{l}$ (11), bei der Ratte um $3000 \mathrm{mOsmol} / \mathrm{l}$ (12), beim Schwein $1000 \mathrm{mOsmol} / l(13)$.

Wenn eine Beziehung zwischen Mucopolysaccharidgehalt und maximal möglicher Osmolarität des Harns besteht, sollte die Mucopolysaccharidkonzentration eine charakteristische Verteilung in einzelnen, anatomisch definierten Nierenzonen besitzen und bei einzelnen Spezies, die in unterschiedlichem Maße Harn konzentrieren können, entsprechend dieser Fähigkeit variieren.

\section{Material und Methoden}

\section{Untersucbungsmaterial}

Von 650 Albinoratten (Körpergewicht $180-250 \mathrm{~g}$ ) wurden beide Nieren in Äther-Sauerstoff-Narkose entnommen. Die Entnahme der Nieren von 13 Hunden (2 Bastarde, 11 Beagle; 1,5-2,5 Jahre) und von 20 Schweinen (Schlachthofmaterial) erfolgte unmittelbar post mortem. Die Nieren wurden dekapsuliert und die Nierenpapillen mit angrenzendem äußeren Nierenmark und Nierenrinde präpariett. Die Nierenpapille wurde von der Spitze zur Basis hin in folgender Weise längs unterteilt: Bei der Ratte stellt der erste Abschnitt mit 1-1,5 mm die Papillenspitze dar, das folgende $2 \mathrm{~mm}$ lange Segment ist der untere Anteil des inneren Markes. Der anschließende obere Anteil des inneren Markes besitzt eine Länge von $1-1,5 \mathrm{~mm}$. Beim Hund ist die Papillenspitze $2 \mathrm{~mm}$, der untere Anteil des inneren Markes 4-5 mm und der obere Anteil des inñeren Markes $2 \mathrm{~mm}$ làng. Beim Schwein besitzen die Segmente der Papille eine Länge von je 1-1,5 mm. Beim Ptäparieren des äußeren Nierenmarkes wurde bei allen Tieren eine schmale Zone an der Mark-Rinden-Grenze verworfen. Von der Nierenrinde wurde nur eine schmale subcapsuläre Zone zur Analyse entnommen. Die Grenze zwischen innerem Mark oben und äußerem Mark ist bei der Schweineniere unscharf, so daß eine exakte Zonentrennung zum Teil kaum möglich war. Alle Präparate wurden in Aceton getrocknet.

\section{Analysen}

Der Wassergehalt des Gewebes wurde durch Aceton- bzw. Gefriertrocknung bestimmt. Bei 2-3 Serien wurde nach der Zonentrennung das Frischgewicht ermittelt und die Trocknung bis zur Gewichtskonstanz durchgeführt. Um die Verdunstung von der Entnahme des Gewebes bis zum Wägen zu berücksichtigen, wurden in Abhängigkeit rom Gewicht des Gewebsstückes Verdunstungskurven aufgenommen. Das wahre Feuchtgewicht wurde durch Retropolation auf die Entnahmezeit ermittelt.

Die Bestimmung der löslichen und strukturgebundenen DNA erfolgte nach 1. c. (14). In allen Nierenzonen beträgt der Anteil der löslichen DNA etwa 10\% der strukturgebundenen DNA.
Hexosamin (15), Uronsäure (16), Sulfat (17) und Hydroxyprolin (18) wurden nach früher beschriebenen Methoden bestimmt. Zur Hexosaminbestimmung im Trockengewebe wurden 10-12 mg Material mit $5 \mathrm{ml} 5 \mathrm{~N} \mathrm{HCl} 5 \mathrm{Stdn}$. bei $110^{\circ}$ in unter Stickstoff verschlossenen Ampullen hydrolysiert und im Hydrolysat nach Filtration die Elson-Morgan-Reaktion durchgeführt.

Aus dem Hydroxyprolingehalt errechnet sich durch Multiplikation mit dem Faktor 7,46 der Kollagengehalt (19). Die Bestimmung von Glucosamin und Galaktosamin erfolgte kolorimetrisch nach 1. c. (20).

\section{Präparative Darstellung saurer Mucopolysaccharide}

Das nach topographischen Kriterien erhaltene acetongetrocknete Material wurde gleichzeitig und unter identischen Bedingungen proteolytisch aufgeschlossen. $100 \mathrm{mg}$ Trockengewebe wurden in $1 \mathrm{ml}$ eines Cystein-aktivierten Papain-Puffergemisches (0,5\% Papain, Merck Nr. 7147, 0,1 M Acetatpuffer pH 6,0) 96 Stdn. unter dreimaliger Papainzugabe bei $60^{\circ}$ inkubiert. Zur Untersuchung gelangten je nach Menge des Ausgangsmaterials $270-2200 \mathrm{mg}$ Gewebe.

Nach Beendigung des proteolytischen Abbaus wurde der nach Zentrifugation bei $74000 \mathrm{~g}$ erhaltene Uberstand in einem $4 \mathrm{bzw}$. $9 \mathrm{~m} /$ fassenden Ultrazentrifugen-Röhrchen mit Cetylpyridiniumchlorid in einer Endkonzentration von 1\% versetzt. Nach $60 \mathrm{Min}$. wurde das Präzipitat durch Zentrifugation bei $74000 \mathrm{~g}$ gewonnen, der Uberstand verworfen und im gleichen Röhrchen bei Vorliegen größerer Proteolysatmengen erneut eine CetylpyridiniumchloridFällung vorgenommen. Das gesammelte, mit Cetylpyridiniumchlorid gefällte Material wurde in $1,5 \mathrm{~N} \mathrm{MgCl}_{2}$ gelöst, mit 2,5 Vol. Åthanol und Kaliumacetat (1\% Endkonzentration) gefällt, das Präzipitat $3 \mathrm{mal}$ mit Äthanol gewaschen und mit Diäthyläther getrocknet.

\section{Trennung sautrer Mucopolysaccharide}

War die Menge an dargestellten sauren Mucopolysacchariden ausreichend groß, wurde eine Trennung in einzelne Mucopolysaccharidfraktionen über die Cetylpyridiniumkomplexe vorgenommen. $10-12 \mathrm{mg}$ Material wurden in $2 \mathrm{~m} /$ dest. Wasser gelöst, mit $2 \mathrm{~m} /$ 2proz. Cetylpyridiniumchlorid in $0,02 \mathrm{~N} \mathrm{MgCl}_{2}$ versetzt, der nach Zentrifugation bei $105000 \mathrm{~g}$ erhaltene Niederschlag mit $0,25 \mathrm{~N}$ $\mathrm{MgCl}_{2}$ fein verrührt, der Uberstand nach erneutem Zentrifugieren mit 2,5 Vol. Äthanol und 1\% Kaliumacetat (Endkonzentration) gefällt, gewaschen und getrocknet. Der Rückstand wurde mit $0,5 \mathrm{~N} \mathrm{MgCl}_{2}$ bzw. nach nochmaligem Zentrifugieren mit $1,5 \mathrm{~N}$ $\mathrm{MgCl}_{2}$ versetzt und die erhaltenen Úberstände wie beschrieben behandelt.

Die elektrophoretische Auftrennung der sauren Mucopolysaccharide sowie die Durchführung des Hyaluronidaseabbaus erfolgte nach 1. c. (21).

\section{Ergebnisse}

Der Wassergehalt des Nierengewebes ist bei allen untersuchten Spezies in der Nierenpapille am größten (Tab. 1). Zur Rinde hin erfolgt eine Abnahme des Wassergehaltes. Nach Acetontrocknung ist bei Ratte und Hund das proz. Trockengewicht etwas niedriger als nach Gefriertrocknung. Beim Schwein ergeben Aceton- und Gefriertrocknung etwa gleiche Werte.

Der Kollagengehalt des Frischgewebes wurde aus der Hydroxyprolinanalyse des Trockengewebes unter Berücksichtigung des Wassergehaltes ermittelt (Abb. 1). Bei Hund und Schwein ändert sich der Kollagengehalt in den verschiedenen Nierenzonen in ähnlicher Weise. Die höchste Konzentration an Kollagen findet sich im inneren Mark oben. Zur Nierenrinde hin nimmt der Kollagengehalt stark ab. Im Gegensatz dazu ist der Kollagengehalt der Rattenniere sehr gering und zeigt in den einzelnen Zonen kaum Unterschiede. 
Aus der Hexosaminanalyse ergeben sich keine auffälligen Unterschiede im Aminozuckergehalt von Hunde-, Ratten- und Schweinenieren (Tab. 1). Bei allen Tieren ist der Hexosamingehalt in der Nierenpapille höher als im äußeren Mark und in der Nierenrinde. Die im Frischbzw. Trockengewebe ermittelte Konzentration an Aminozuckern stellt die Summe der in Mucopolysacchariden und Glykoproteinen enthaltenen Aminozucker dar. Wird mit Hilfe der Hexosaminanalyse der präparativ dargestellten Mucopolysaccharide der Gesamtgehalt an Aminozuckern auf Glykoprotein-Hexosamin und Mucopolysaccharid-Hexosamin aufgeschlüsselt, ergeben sich dagegen auffällige Speziesunterschiede. Beim Hund stammen bis zu $40 \%$ des Gesamthexosamins aus sauren Mucopolysacchariden, bei Ratte und Schwein lediglich bis zu $20 \%$. Die Nierentinde aller Tierarten enthält überwiegend Aminozucker aus Glykoproteinen.

Analytische Daten über die dargestellten Mucopolysaccharide enthält Tabelle 2 . Aus ihnen läßt sich unter Berücksichtigung der für Reinsubstanzen erhaltenen Hexosamin- und Uronsäureanalysen und des Wassergehalts die Mucopolysaccharidkonzentration im Frischgewebe berechnen (Abb. 2). Das innere Mark unten - die bei Ratte und Hund ausgedehnteste Zone der

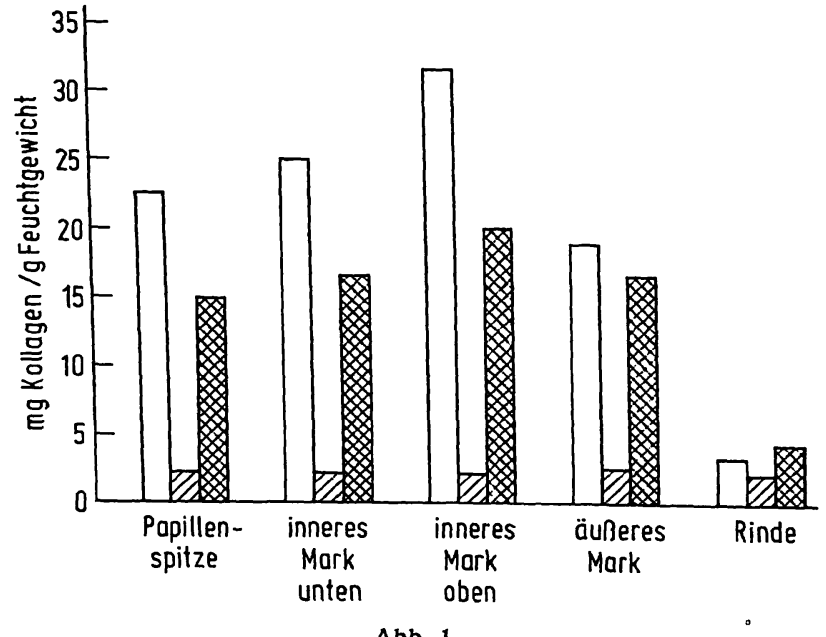

Kollagengehalt in verschiedenen topographischen Zonen der Niere von Hund (offene Säulen), Ratte (schraffierte Säulen) und Schwein
(doppelt schraffierte Säulen)
Tab. 1

Anteil des Acetontrockengewebes (TG) am Frischgewebe in verschiedenen topographischen Regionen von Hunde-, Ratten- und Schweinenieren. Hexosamingehalt (HexN) des Trockengewebes und prozentuale Verteilung der Aminozucker auf saure Mucopolysaccharide (sMPS) und Glykoproteine, errechnet mit Hilfe von Tab. 2. Gesamt-Hexosamingehalt $=100 \%$

\begin{tabular}{|c|c|c|c|c|c|c|c|c|c|c|c|c|}
\hline & $\begin{array}{c}\text { mg TG/g } \\
\text { Frisch- } \\
\text { gewebe }\end{array}$ & $\begin{array}{c}\text { H } \\
\text { nMol } \\
\text { HexN/ } \\
\text { mg TG }\end{array}$ & $\begin{array}{l}\text { und } \\
\% \text { sMPS- } \\
\text { HexN }\end{array}$ & $\begin{array}{l}\text { \% Glyko- } \\
\text { protein- } \\
\text { HexN }\end{array}$ & $\begin{array}{c}\text { mg TG/g } \\
\text { Frisch- } \\
\text { gewebe }\end{array}$ & 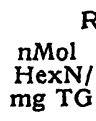 & $\begin{array}{l}\text { atte } \\
\% \text { sMPS- } \\
\text { HexN }\end{array}$ & $\begin{array}{l}\text { \% Glyko- } \\
\text { protein- } \\
\text { HexN }\end{array}$ & $\begin{array}{c}\text { mg TG/g } \\
\text { Frisch- } \\
\text { gewebe }\end{array}$ & $\begin{array}{l}\text { Sch } \\
\text { nMol } \\
\text { HexN/ } \\
\text { mg TG }\end{array}$ & $\begin{array}{l}\text { wein } \\
\% \text { sMPS- } \\
\text { HexN }\end{array}$ & $\begin{array}{l}\text { \% Glyko- } \\
\text { protein- } \\
\text { HexN }\end{array}$ \\
\hline $\begin{array}{l}\text { Papillenspitze } \\
\text { Inneres Mark } \\
\text { unten }\end{array}$ & $\begin{array}{l}143 \\
154\end{array}$ & $\begin{array}{l}69,3 \\
79,9\end{array}$ & $\begin{array}{l}22 \\
40\end{array}$ & $\begin{array}{l}78 \\
60\end{array}$ & $\begin{array}{l}182 \\
141\end{array}$ & $\begin{array}{l}91,6 \\
78,8\end{array}$ & $\begin{array}{r}8 \\
20\end{array}$ & $\begin{array}{l}92 \\
80\end{array}$ & $\begin{array}{l}154 \\
166\end{array}$ & $\begin{array}{l}81,6 \\
87,2\end{array}$ & $\begin{array}{l}16 \\
20\end{array}$ & $\begin{array}{l}84 \\
80\end{array}$ \\
\hline Inneres Mark & 150 & 77,7 & 41 & 59 & 138 & 82,7 & 16 & 84 & 171 & 82,7 & 11 & 89 \\
\hline $\begin{array}{l}\text { Äußeres Mark : : } \\
\text { Rinde }\end{array}$ & $\begin{array}{l}191 \\
224\end{array}$ & $\begin{array}{l}56,4 \\
53,6\end{array}$ & $\begin{array}{r}21 \\
6\end{array}$ & $\begin{array}{l}79 \\
94\end{array}$ & $\begin{array}{l}206 \\
259\end{array}$ & $\begin{array}{l}48,6 \\
55,9\end{array}$ & $\begin{array}{r}11 \\
3\end{array}$ & $\begin{array}{l}89 \\
97\end{array}$ & $\begin{array}{l}181 \\
236\end{array}$ & $\begin{array}{l}85,5 \\
59,8\end{array}$ & $\begin{array}{l}8 \\
7\end{array}$ & $\begin{array}{l}92 \\
93\end{array}$ \\
\hline
\end{tabular}

Tab. 2

Gehalt des Acetontrockengewebes (TG) verschiedener topographischer Regionen von Hunde-, Ratten- und Schweinenieren an sauren Mucopolysacchariden (SMPS), repräsentiert durch Hexosamin-, Uronsäure- (GUA) und Sulfatanalyse präparativ dargestellter sMPS. Elektrophoretische Auftrennung der Gesamt-Mucopolysaccharide in einzelne Mucopolysaccharidtypen (HA $=$ Hyaluronsäure, HS $=$ Heparitinsulfat, DS $=$ Dermatansulfat, $\mathrm{CS}=$ Chondroitinsulfat)

\begin{tabular}{|c|c|c|c|c|c|c|c|c|c|c|c|c|c|c|c|}
\hline & \multicolumn{5}{|c|}{ Hund } & \multicolumn{5}{|c|}{ Ratte } & \multicolumn{5}{|c|}{ Schwein } \\
\hline & 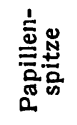 & 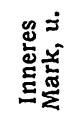 & @゚ & 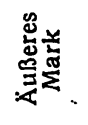 & 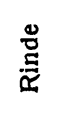 & 总罢 & 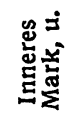 & 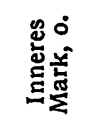 & 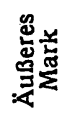 & $\underset{\partial}{\stackrel{\partial}{a}}$ & 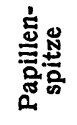 & 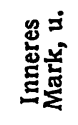 & 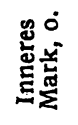 & 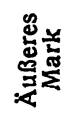 & $\underset{\mathbb{Z}}{\mathbb{\alpha}}$ \\
\hline $\begin{array}{l}\text { nMol Hexosamin/ } \\
\text { mg TG }\end{array}$ & 15,1 & 31,9 & 31,9 & 11,5 & 3,1 & 7,3 & 16,0 & 13,7 & 5,2 & 1,9 & 13,4 & 17,2 & 9,3 & 6,7 & 4,1 \\
\hline nMol GUA/ & 15,4 & 32,8 & 33,0 & 11,3 & 3,6 & 7,5 & 16,0 & $.13,7$ & 5,4 & 1,9 & 12,9 & 17,1 & 9,3 & 6,9 & 4,2 \\
\hline $\begin{array}{c}\text { nMol SOal } \\
\text { mg TG }\end{array}$ & 9,2 & 12,1 & 14,3 & 8,5 & 2,8 & 7,2 & 10,1 & 9,1 & 3,5 & 1,6 & 6,7 & 9,2 & 6,3 & 6,1 & 3,1 \\
\hline $\begin{array}{ll}\text { mg sMPS- } & \text { HA } \\
\text { Typ/100 mg HS } \\
\text { Gesamt- } & \text { DS } \\
\text { sMPS } & \text { CS }\end{array}$ & $\begin{array}{r}30 \\
9 \\
12 \\
49\end{array}$ & $\begin{array}{l}59 \\
14 \\
10 \\
17\end{array}$ & $\begin{array}{l}48 \\
10 \\
19 \\
23\end{array}$ & $\begin{array}{l}25 \\
15 \\
29 \\
31\end{array}$ & $\begin{array}{l}13 \\
38 \\
49 \\
-\end{array}$ & $\begin{array}{l}\overline{19} \\
26 \\
55\end{array}$ & $\begin{array}{l}33 \\
16 \\
20 \\
31\end{array}$ & $\begin{array}{l}34 \\
14 \\
23 \\
29\end{array}$ & $\begin{array}{l}30 \\
13 \\
57 \\
-\end{array}$ & $\begin{array}{l}14 \\
25 \\
61 \\
-\end{array}$ & $\begin{array}{l}40 \\
17 \\
17 \\
26\end{array}$ & $\begin{array}{l}39 \\
18 \\
19 \\
24\end{array}$ & $\begin{array}{l}26 \\
23 \\
27 \\
24\end{array}$ & $\begin{array}{r}8 \\
25 \\
37 \\
30\end{array}$ & $\begin{array}{l}18 \\
20 \\
62 \\
-\end{array}$ \\
\hline
\end{tabular}

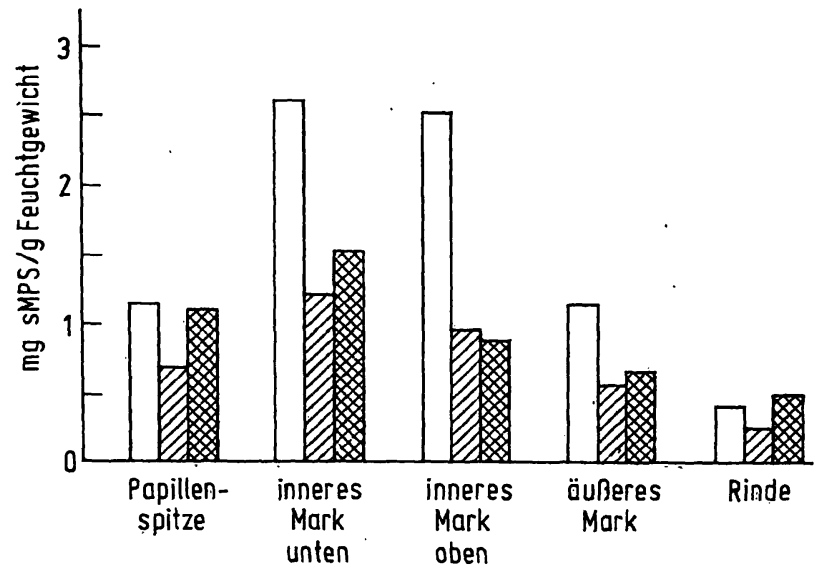

Abb. 2

Mucopolysaccharidgehalt (sMPS) in verschiedenen topographischen Zonen der Niere von Hund (offene Säulen), Ratte (schraffierte Säulen) und Schwein (doppelt schräffierte Säulen) 
gesamten Papille - enthält bei allen drei Tierarten die höchste Konzentration an sauren Mucopolysacchariden. Zur Nierenrinde erfolgt eine stete Abnahme. Der absolute Mucopolysaccharidgehalt ist beim Hund am größten (bis $2,6 \mathrm{mg} / \mathrm{g}$ Feuchtgewicht) und bei der Ratte am geringsten (bis $1,2 \mathrm{mg} / \mathrm{g}$ Feuchtgewicht). Der Mucopolysaccharidgehalt der Scḩweineniere liegt im inneren Mark oben gering unter dem der Rattenniere.

Durch elektrophoretische Trennung lassen sich an einzelnen Polysaccharidtypen Hyaluronsäure, Heparitinsulfat, Dermatansulfat und Chondroitinsulfat nachweisen. Ihr Verteilungsmuster variiert in den verschiedenen Nierenzonen und bei den einzelnen Tieren. Der relative Dermatansulfatgehalt nimmt zur Nierenrinde hin deutlich zu, Chondroitinsulfat fehlt stets in der Nierenrinde. Die Hundeniere zeichnet sich durch hohe Hyaluronsäurekonzentration aus.

Die Ergebnisse der elektrophoretischen Auftrennung werden durch die Mucopolysaccharidfraktionierung über die Cetylpyridiniumkomplexe und die Bestimmung des Galaktosamin-Glucosamin-Verhältnisses gestützt.

Die vorwiegend Chondroitinsulfat enthaltende Cetylpyridiniumchlorid-Fraktion $\mathrm{V}$.(Elution mit $1,5 \mathrm{~N} \mathrm{MgCl}_{2}$ ) aus dem inneren Mark unten von Hund und Ratte ergibt nach Hyaluronidaseabbau im Vergleich mit einem Referenzpräparat aus Chondroitin-6-sulfat eine nicht meßbare Farbreaktion bei der Morgan-Elson-Reaktion. Daraus ist zu schließen, daß die Chondroitinsulfatkomponente zumindest überwiegend Chondroitin-4sulfat darstellt (22). Nach Hyaluronidaseabbau und anschließender Dialyse ist die Chondroitinsulfatfraktion elektrophoretisch nicht mehr nachweisbar.

Das Ergebnis der DNA-Analyse ist aus Abbildung 3 ersichtlich. Die Ratte besitzt in allen Zonen mit Aus-

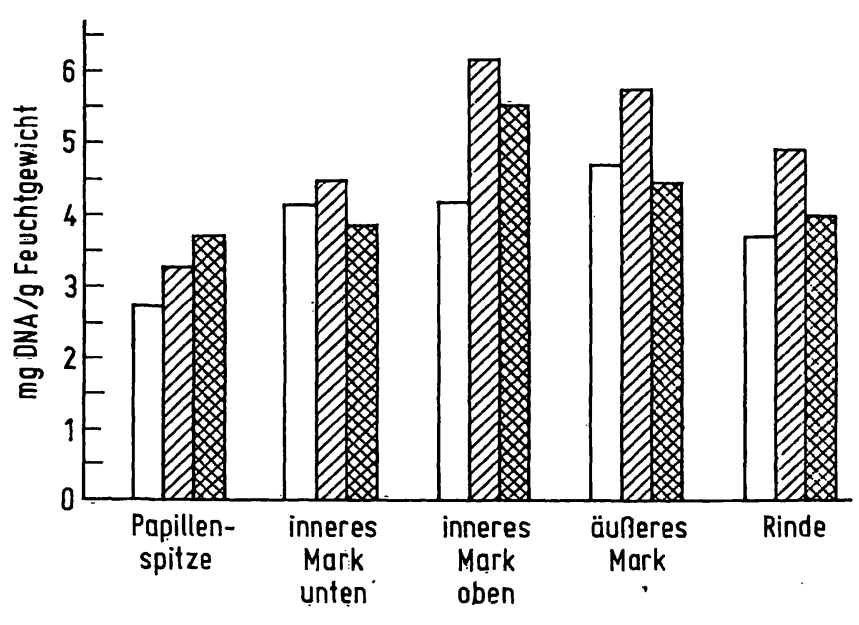

Abb. 3

Gehalt an strukturgebundener DNA in verschiedenen topographischen Zonen der Niere von Hund (offene Säulen), Ratte (schraffierte Säulen) und Schwein (doppelt schraffierte Säulen)

nahme der Papillenspitze den höchsten DNA-Gehalt. Die Werte beim Schwein liegen teilweise über, teilweise unter den Werten des Hundes. Die Papillenspitze enthält den geringsten, das innere Mark oben (Ratte, Schwein) bzw. das äußere Mark (Hund) den höchsten Gehalt an DNA.

\section{Diskussion}

Die Unterteilung des Nierengewebes in verschiedene Zonen basiert auf vergleichenden histochemischen und elektronenmikroskopischen Untersuchungen (5). Die hier dargestellten Ergebnisse bilden ein biochemisches Korrelat zu diesen Befunden.

Aus unseren vergleichenden Untersuchungen ergibt sich, daß bei Hund, Ratte und Schwein der höchste Mucopolysaccharidgehalt in der Nierenpapille - insbesondere in der Zone ,inneres Mark unten“ - gefunden wird. Das äußere Mark besitzt einen niedrigeren Mucopolysaccharidgehalt, in der Nierenrinde wird eine weitere Abnahme beobachtet.

Ähnliche topographische Differenzen ergeben sich aus der Bestimmung des Kollagengehaltes nur für Hund und Schwein. Bei der Ratte ist dagegen die Kollagenkonzentration in allen Zonen etwa gleich.

Dicker und FrankLin (23) fanden bei Hund, Schwein und Schaf keine Unterschiede im Mucopolysaccharidgehalt von Nierenrinde und Nierenmark und zwischen einzelnen Tieren. Der von uns im Vergleich zu den Ergebnissen von DiCker und FrankLIN ermittelte niedrigere Mucopolysaccharidgehalt in der Nierenrinde und das Fehlen von Chondroitinsulfat in dieser Zone könnte durch unterschiedliche Präparation und Zonentrennung erklärt werden. Zur Untersuchung gelangte nur von größeren Gefäßen freies Gewebe. In der Gefäßwand sind insbesondere Chondroitinsulfat und Hyaluronsäure in hoher Konzentration vorhanden. Die bei der Analyse miterfaßten Basalmembranen enthalten keine sauren Mucopolysaccharide (24). Die vorgelegten Ergebnisse der Mucopolysaccharidanalyse stehen dagegen in guter Übereinstimmung mit Befunden von Castor und Greene (25) an der Hundeniere. Diesen Autoren gelang allerdings nicht - im Gegensatz zu 1. c. (23) - der Nachweis von Chondroitinsulfat im Nierenmark. Doch kann an der Existenz von Chondroitinsulfat auf Grund von elektrophoretischer Beweglichkeit und Hyaluronidasesensitivität kein Zweifel bestehen. Differenzen im Verteilungsmuster von Dermatansulfat und Heparitinsulfat bedürfen weiterer $\mathrm{Klä-}$ rung.

Vergleicht man den Mucopolysaccharidgehalt der Nierenpapille der drei Tiere, so ergibt sich, daß beim Hund die Mucopolysaccharidkonzentration am höchsten und bei der Ratte mit Ausnahme des Abschnittes, ,inneres Mark oben" am geringsten ist. Die Nierenpapille des Schweines liegt im Mucopolysaccharidgehalt teilweise zwischen Hund und Ratte. Für die funktionelle Betrachtung ist allerdings die interstitielle Gewebskonzentration allein von Bedeutung. Bei annähernd gleicher Zellgröße innerhalb einzelner entsprechender Zonen deuten speziesabhängige Unterschiede im DNA-Gehalt auf verschiedene Relationen von extra- und intrazellulärem Kompartiment. Da die Rattenniere die höchste DNAKonzentration besitzt, wird bei der Ratte der interstitielle Mucopolysaccharidgehalt gegenüber Hund und Schwein relativ größer. 
Für die Funktion der sauren Mucopolysaccharide, als Polyelektrolyte Ionen und Wasser zu binden, ist neben ihrem Verteilungsmuster die Anzahl freier anionischer Gruppen und das Molekulargewicht der PolysaccharidProteine von Bedeutung.

Durch Interaktion zwischen anionischen Gruppen der sauren Mucopolysaccharide und kationischen Gruppen des Kollagens (26) oder anderer Proteine kann ein Teil der Anionen maskiert werden. $\mathrm{Da}$ die Nierenpapille von Schwein und Hund 10 bis $15 \mathrm{mal}$ soviel Kollagen enthält wie die Rattennierenpapille, könnte dieser Effekt bei Hund und Schwein stärker als bei der Ratte sein. Eine Ionenbilanz ist aus den vorliegenden Daten jedoch nicht möglich, da nur ein kleiner Teil der Trockensubstanz analytisch erfaßt wurde und da die Tertiärstruktur des Kollagens eine quantitative Betrachtung der Ladungsverhältnisse unmöglich macht.

Bei hohem Turnover der Mucopolysaccharide kann durch Änderung des Molekulargewichtes die Wasserbindungskapazität erheblich variieren. Die Absoluthöhe der Mucopolysaccharidkonzentration wäre dabei gegenüber dem Stoffumsatz von geringerer Bedeutung.

Zum anderen besteht die Möglichkeit, daß Mucopolysaccharide in ihrer Verbindung mit Protein unter Ein- schluß des Lösungswassers ein dichtes Gel bilden. Da das Wasser dann nicht frei austauschbar ist, entstünde eine Permeationsschranke, deren Wirksamkeit durch das Vorhandensein von Faserproteinen noch erhöht würde. Diese Behinderung der freien Diffusion wäre bei der Ratte auf Grund des niedrigen Mucopolysaccharid- und Kollagengehaltes im Gegensatz 'zu Hund und Schwein von geringerer Bedeutung. Hinzu kommt, da $B$ in der Rattenniere die niedrigste Konzentration an Hyaluronsäure, die ein besonders großes Wasserbindungsvermögen besitzt, gefunden wurde. Es wurde außerdem festgestellt, daß beim Hund das Hyaluronat des Nierenmarkes ein höheres Molekulargewicht und damit ein größeres hydrodynamisches Volumen besitzt als das der Nierentinde (25).

Es bestehen somit zwei Möglichkeiten, wie die sauren Mucopolysaccharide im Zusammenspiel mit Faserproteinen im Interstitium der Nierenpapille die Harnkonzentrierung beeinflussen können: einmal, indem sie als Polyelektrolyte Ionen und Wasser reversibel binden und Konzentrationsunterschiede schaffen, zum anderen, indem sie als Permeationsschranke die freie Diffusion beeinflussen.

\section{Literatur}

1. MöllendorfF, W. von, Handbuch der Mikroskopischen Anatomie des Menschen, Bd. VII, S. 140, Springer-Verlag, Berlin (1930). - 2. LApP, H. und A. Nolte, Frankf. Zschr. Path. 71, 617 (1962). - 3. Rinehart, J. F. und S. K. Abul-Hay, Arch. Path. Chicago 52, 189 (1951); Geyer, G., H. Feuerstein und E. GrunDIG, Acta histochemica 18, 186 (1964). - 4. Abrahams, C. und C. L. Pirani, S. Afr. J. Med. Sc. 31, 107 (1966). - 5. Grossmann, A., in Vorbereitung. - 6. Farber, S. J., Circulation 21, 941 (1960). -7. Buddecke, E., W. KRöz und W. Trtror, Hoppe-Seyler's Z. physiol. Chem. 348, 651 (1967). - 8. Nolte, A., Verh. dtsch. Ges. Pathol., 51. Tagung, 316 (1967). - 9. Pinter, G. G., Experientia Basel 23, 100 (1967). - 10. Trump, B. F. und R. E. Bulger, Morphology of the Kidney; In: Structural Basis of Renal Disease. Ed. E. Lovell Becher, Harper und Row, New York (1968). UlLRICH, K. J., Japanese Society of Nephrology, Tokio, Abstracts of the 8th annual Meeting, 8 Nr. 1, (1966). - 11. ULLRICH, K. J. und K. H. Jarausch, Pflügers Arch. Physiol. 262, 537 (1956). - 12. Lilien, O. M., J. Urol., Baltimore 95, 90 (1966). — 13. Sснмidt-
Nielsen, B., Physiol. Rev., Baltimore 38, 139 (1958). - 14. WebB, J. und H. B. Levy, J. biol. Chemistry 213, 107 (1955). 15. BoAs, N., J. biol. Chemistry, 204, 553 (1953). - 16. Brrter, T. und H. MUIR, Analytic. Biochem. 4, 330 (1962). - 17. SîturSATZ, H. W., Dissertation, Technische Hochschule Aachen, (1963). - 18. Stegemann, H., Hoppe-Seyler's Z. physiol. Chem. 311, 41 (1958). - 19. NeumanN, R. E. und M. A. Logan, J. biol. Chemistry 184, 299 (1950). - 20. LuDowIEG, J. und U. D. BENMAMAN, Analytic Biochem. 19, 80 (1967). - 21. Kresse, H. und E. BupDECKE, Hoppe-Seyler's Z. physiol. Chem. 351, 151 (1970). MANLEY, G., R. N. Mullinger und P. H. Lloyp, Biochem. J. 114, 89 (1969). - 22. Buddecke, E. und D. Platt, Hoppe-Seyler's Z. physiol. Chem. 343, 61 (1965). - 23. Dicker, S. E. und C. S. FrankLin, J. Physiol. 186, 110 (1966). - 24. SpIro, R. G., J. biol. Chemistry 242, 1915 (1967). - 25. Castor, C. W. und J. A. Greene, J. Clin. Invest. 47, 2125 (1968). - 26. MAthews, M. B., Biochem. J. 96, 710 (1965). 